Research Article

\title{
Global Stability for a Semidiscrete Logistic System with Feedback Control
}

\author{
Li Xu $\mathbb{D}^{1,2}$ Shanshan Lou, ${ }^{2}$ and Ruiwen Han $^{2}$ \\ ${ }^{1}$ School of Mathematics, Tianjin University, Tianjin 300072, China \\ ${ }^{2}$ School of Science, Tianjin University of Commerce, Tianjin 300134, China \\ Correspondence should be addressed to Li Xu; beifang_xl@163.com
}

Received 20 April 2020; Revised 10 June 2020; Accepted 3 July 2020; Published 24 July 2020

Guest Editor: Tarek F. Ibrahim

Copyright (c) $2020 \mathrm{Li} \mathrm{Xu}$ et al. This is an open access article distributed under the Creative Commons Attribution License, which permits unrestricted use, distribution, and reproduction in any medium, provided the original work is properly cited.

In this paper, a semidiscrete logistic model with the Dirichlet boundary conditions and feedback controls is proposed. By means of the sub- and supper-solution method and eigenvalue theory, the unique positive equilibrium is proved. By constructing a suitable Lyapunov function, the global asymptomatic stability of the unique positive equilibrium is investigated. Finally, numerical simulations are presented to verify the effectiveness of the main results.

\section{Introduction}

The logistic model has been widely studied; its importance arises because it describes the typical behavior of many dynamic systems modeled by nonlinear differential equations [1-5]. Applications involve different areas, including biology, mathematics, engineering, physics, and many others, to forecast and describe the dynamic changing process and trend of the matters. The standard logistic population model of single species can be described in the following form:

$$
\frac{\mathrm{d} x}{\mathrm{~d} t}=r x(K-x),
$$

where $x(t)$ is the population size at the time $t, r$ the intrinsic growth rate, and $K$ the carrying capacity of environment. Model (1) can also be written in the following form:

$$
\frac{\mathrm{d} x}{\mathrm{~d} t}=x(r-a x)
$$

It is well known that spatial heterogeneity and dispersal play an important role in the dynamics of populations, such as the role of dispersal in the maintenance of patchiness, or spatial population variation [6-9]; then, space can be taken into account in all fundamental aspects of ecological organization. We have to notice that the motion of individuals is random and isotropic, i.e., without any preferred direction, and the individuals are also absolute ones in microscopic sense, and each isolated individual exchanges materials by diffusion with its neighbors $[10,11]$. Thus, it is reasonable to consider a $1 \mathrm{D}$ continuous time but discrete space logistic model as follows:

$$
\frac{\mathrm{d} x_{i}(t)}{\mathrm{d} t}=x_{i}(t)\left(r-a x_{i}(t)\right)+D \nabla^{2} x_{i-1}(t),
$$

with the discrete Dirichlet boundary conditions,

$$
x_{0}(t)=0=x_{n+1}(t),
$$

where $D$ is the diffusion coefficient, $i \in[1, n]=\{1,2$, $\ldots, n\}, t \in R^{+}$, and $\nabla^{2}$ is discrete Laplace operator:

$$
\nabla^{2} x_{i-1}(t)=x_{i+1}(t)-2 x_{i}(t)+x_{i-1}(t)
$$

It is a fact that ecosystem in the real world is continuously distributed by unpredictable forces which may result into changes in the biological parameters such as survival rates [12]. Whether the ecosystem can withstand those unpredictable forces which persist for a finite period of time is worthy of consideration from the practical interest in ecology. On the contrary, the diffusion-driven instability may emerge if the steady state solution is stable to small 
spatial perturbations in absence of diffusion, but unstable when diffusion is present [6]. If the diffusion-driven instability should be avoided in some situations, and system parameters are not easy to adjust, then some other ways should be adopted to achieve the stabilization aim [13]. There may exist a situation where the equilibrium of the dynamical model is not the desirable one (or affordable) and a smaller value of the equilibrium is required. Then, altering the model structure so as to make the population stabilize at a lower value is necessary [14].

In these situations, feedback control will be an effective way to alter the positions of positive equilibrium or to obtain its stability. To achieve the aim, one of the techniques used is to alter system structurally by introducing "indirect control" variables [15]. Then, the control variables can be added into systems (3) and (4); the following continuous time but discrete space logistic model with feedback control can be obtained:

$$
\left\{\begin{array}{l}
\frac{\mathrm{d} x_{i}(t)}{\mathrm{d} t}=x_{i}(t)\left(r-a x_{i}(t)-b u_{i}(t)\right)+D \nabla^{2} x_{i-1}(t), \\
\frac{\mathrm{d} u_{i}(t)}{\mathrm{d} t}=-\eta u_{i}(t)+e x_{i}(t)
\end{array}\right.
$$

with the discrete Dirichlet boundary conditions,

$$
x_{0}(t)=0=x_{n+1}(t) .
$$

During the last years, many scholars did works on the continuous time Logistic model and related system with feedback controls (see, e.g., [16-22] and references cited therein). In 1992, Gopalsamy introduced a certain differential equation as a feedback control variable into the logistic models and discussed the asymptotic behavior of solutions for the model with feedback controls [16]. The global asymptotical stability of a logistic equation with feedback control was considered by means of a method combined with the upper and lower solution technique in [17]. In [18], influence of dispersal and strong Allee effect on a two-patch predator-prey model was studied and existence and stability criterion of the interior equilibrium point of the system was analyzed in presence as well as in absence of dispersal speed. Feedback control strategy was introduced into an SI epidemic model, the effect of population migration among $n(n \geq 2)$ different patches was considered, and globally asymptotically stable conditions for the unique endemic equilibrium was given by means of the method of global Lyapunov functions and results from graph theory [22]. To the best of the authors' knowledge, to this day, still no scholar has investigated the global asymptomatic stability of the continuous time but the discrete space logistic model with the discrete Dirichlet boundary conditions and feedback control.

The organization of this paper is as follows. In Section 2, some assumptions and preparations are given, according to the sub- and supper-solution method, and the unique positive equilibrium of systems (6) and (7) is proved. Then, the asymptotically stability of the unique positive equilibrium is proved by constructing a nonnegative Lyapunov function without the results from graph theory in Section 3. In Section 4, numerical simulations are presented to illustrate the feasibility of our main results. In Section 5, a brief discussion is given.

\section{Preliminaries}

From the view point of biology, we only need to discuss the positive solution of systems (5) and (6). So, it is assumed that the initial conditions of (6) and (7) are of the form

$$
\begin{aligned}
& x_{i}(0)>0 \text {, } \\
& u_{i}(0)>0 \text {, } \\
& i=1,2, \ldots, n \text {. }
\end{aligned}
$$

For our purpose, we first introduce the following lemma, which can be obtained easily by comparison theorem of ODE.

Lemma 1. Any positive solution $x(t)$ of the inequality problem

$$
\frac{\mathrm{d} x(t)}{\mathrm{d} t} \leq-a x+b,
$$

satisfies

$$
\lim _{t \rightarrow \infty} \sup x(t) \leq \frac{b}{a}
$$

if $a>0, b>0$.

Now, we are in the position to consider the positivity and boundedness of the solutions for (6) and (7).

Theorem 1. The solution of system (6) and (7) with the initial conditions (8) remains positive and bounded for all $t>0$.

Proof. It is clear that the solutions of systems (6) and (7) with the initial conditions

$$
\begin{aligned}
& x_{i}(0)>0, \\
& u_{i}(0)>0,
\end{aligned}
$$

are positive for all $t>0$.

Next, we prove that the solutions of systems (6) and (7) are bounded:

$$
\begin{aligned}
\sum_{i=1}^{n} \frac{d x_{i}(t)}{d t}+\sum_{i=1}^{n} x_{i}(t)= & \frac{d}{d t}\left(\sum_{i=1}^{n} x_{i}(t)\right)+\sum_{i=1}^{n} x_{i}(t) \\
= & \sum_{i=1}^{n}\left(x_{i}(t)\left(r-a x_{i}(t)-b u_{i}(u)\right)+D \nabla^{2} x_{i-1}(t)\right) \\
& +\sum_{i=1}^{n} x_{i}(t) \\
= & \sum_{i=1}^{n} x_{i}(t)\left(r-a x_{i}(t)-b u_{i}(u)\right)+\sum_{i=1}^{n} x_{i}(t) \\
& -D\left(x_{1}(t)+x_{n}(t)\right) \\
\leq & \sum_{i=1}^{n}(r+1) x_{i}(t)-a x_{i}^{2}(t) \\
= & \sum_{i=1}^{n}\left(-a\left(x_{i}(t)-\frac{r+1}{2 a}\right)^{2}+\frac{(r+1)^{2}}{4 a}\right) \\
\leq & \sum_{i=1}^{n} \frac{(r+1)^{2}}{4 a}=\frac{n(r+1)^{2}}{4 a} .
\end{aligned}
$$

By means of Lemma $1, \quad \lim \sup \sum_{i=1}^{n} x_{i}(t) \leq$ $n(r+1)^{2} / 4 a=A$. This indicates that all $\vec{x}_{i}^{\infty}(t)$ are uniformly ultimately bounded for $i \in[1, n]$. 
Note that the second equation of system (6) implies

$$
\begin{aligned}
u_{i}(t) & =u_{i}(0) \exp \{-\eta t\}+e \exp \{-\eta t\} \int_{0}^{t} \exp \{\eta s\} x_{i}(s) \mathrm{d} s \\
& \leq u_{i}(0) \exp \{-\eta t\}+\frac{e A}{\eta}(1-\exp \{-\eta t\}) \\
& \leq u_{i}(0)+\frac{e A}{\eta} .
\end{aligned}
$$

This concludes the proof of Theorem 1 .

Next, we will consider the uniqueness and existence of nontrivial positive equilibrium solutions system (6) and (7). Firstly, we list three lemmas, which will be useful to establish the main result.

Lemma 2 (see [23]). For eigenvalue problem,

$$
\left\{\begin{array}{l}
\nabla^{2} x_{i-1}=-\lambda x_{i}, \\
x_{0}=0=x_{n+1},
\end{array} \quad i \in[1, n]=\{1,2, \ldots, n\},\right.
$$

there is $\lambda_{k}=4 \sin ^{2}(k \pi / 2(n+1))$, and $\lambda_{1} \leq \lambda_{k} \leq \lambda_{n}$ for all $k \in[1, n]$.

Lemma 3 (see [24]). Consider the following problem:

$$
\begin{cases}-\nabla^{2} x(z)=f(z, x(z)), & z \in \Omega, \\ x(z)=0, & z \in \partial \Omega,\end{cases}
$$

where is $\Omega \subset Z^{N}$ a finite domain and $f$ defined on $\Omega \times R$ is a function continuous in the second variable. Suppose that (15) has a subsolution $\underline{x}$ and a supersolution $\bar{x}$, with $\underline{x} \leq x \leq \bar{x}$. Assume that there exists a constant $k \geq 0$ such that

$$
f\left(z, s_{2}\right)-f\left(z, s_{1}\right) \geq k\left(s_{2}-s_{1}\right),
$$

for all $z \in \Omega$ and $s_{2} \geq s_{1}$ with $s_{1}, s_{2} \in[a, b]$, where $a=\min _{z \in \Omega} x$ and $b=\max _{z \in \Omega} \bar{x}$. Then, there exist solutions $u$ and $v$ for problem (15) such that $x \leq u \leq v \leq \bar{x}$. Moreover, any solution $x$ of (15) with $x \leq x \leq \bar{x}$ satisfies $u \leq x \leq v$.

Lemma 4 (see [24]). Let $f(z, x)=x h(z, x)$. Suppose that there exists a constant $M>0$ such that $h(z, x) \leq 0$ for $z \in \Omega, x \geq M$ and that $h(z, x)$ is smooth strictly decreasing in $x \in[0, M]$. Then, problem (15) admits at most one positive solution.

We have the following result for the system:

$$
\left\{\begin{array}{l}
0=x_{i}\left(r-a x_{i}-b u_{i}\right)+D \nabla^{2} x_{i-1}, \\
0=-\eta u_{i}+e x_{i} .
\end{array}\right.
$$

Theorem 2. System (17) has a unique positive solution if $r>4 D \sin ^{2}(\pi / 2(n+1))$.

Proof. Substituting the second equation into the equation of system (17), it follows that

$$
0=\frac{1}{D} x_{i}\left(r-a x_{i}-\frac{b e}{\eta} x_{i}\right)+\nabla^{2} x_{i-1} .
$$

Then, we only will consider the uniqueness and existence of nontrivial positive equilibrium solutions system (17) with the discrete Dirichlet boundary conditions (7).

By Theorem 1, any nonnegative and nontrivial solution $v$ of (17) must satisfy $0<v \leq B=\sup _{i \in[1, n]}\left\{x_{i}\right\}=B \leq A$ for $i \in[1, n]$, if $t>0$ is given.

For any $u, v \in[0, B]$ with $u \geq v$, we have

$$
\begin{gathered}
\frac{1}{D} u\left(r-\frac{a \eta+b e}{\eta} u\right)-\frac{1}{D} v\left(r-\frac{a \eta+b e}{\eta} v\right) \\
=\frac{1}{D}(u-v)\left(r-\frac{a \eta+b e}{\eta}(u+v)\right) \\
\geq \frac{1}{D}(u-v)\left(r-\frac{2 B(a \eta+b e)}{\eta}\right) \geq 0,
\end{gathered}
$$

if $r-(2 B(a \eta+b e) / \eta) \geq 0$ holds.

Notice that

$$
\lambda_{1}=4 \sin ^{2} \frac{\pi}{2(n+1)},
$$

is the first eigenvalue of (6), and assume that $\varphi^{(1)}$ is the corresponding positive eigenfunction. Then,

$$
\begin{gathered}
-\nabla^{2}\left(\varepsilon \varphi_{i}^{(1)}\right)-\frac{1}{D} \varepsilon \varphi_{i}^{(1)}\left(r-\frac{a \eta+b e}{\eta} \varepsilon \varphi_{i}^{(1)}\right) \\
=\lambda_{1} \varepsilon \varphi_{i}^{(1)}-\frac{1}{D} \varepsilon \varphi_{i}^{(1)}\left(r-\frac{a \eta+b e}{\eta} \varepsilon \varphi_{i}^{(1)}\right) \\
=\varepsilon \varphi_{i}^{(1)}\left(\lambda_{1}-\frac{r}{D}+\frac{a \eta+b e}{\eta D} \varepsilon \varphi_{i}^{(1)}\right)<0 .
\end{gathered}
$$

For enough $\varepsilon>0$ small if $\lambda_{1} D<r$. Thus, $\varepsilon \varphi_{i}^{(1)}$ can be regarded as a subsolution of (17). On the contrary, $B$ can be a supersolution of (17). By using Lemma 2, problem (17) has a positive solution when the condition $r>4 D \sin ^{2}(\pi / 2(n+$ 1)) holds.

In view of Lemma 3, we let

$$
f(u)=u\left(r-\frac{a \eta+b e}{\eta} u\right)=u h(u),
$$

and there exists a constant $M=(r \eta /(a \eta+b e))>0$ such that $h(u) \leq 0$ for $u \in \Omega, u \geq M$, and that $h(u)$ is smooth strictly decreasing in $u \in[0, M]$. On the basis of Lemma 3, problem (17) admits at most one positive solution. The proof is finished.

\section{Global Stability of Positive Equilibrium}

In this section, we devote ourselves to studying the global asymptotic stability of the unique positive equilibrium $E^{*}\left(x_{1}^{*}, x_{2}^{*}, \ldots, x_{n}^{*}, u_{1}^{*}, u_{2}^{*}, \ldots, u_{n}^{*}\right)$. By using global Lyapunov function, we derive the sufficient conditions under which the positive equilibrium is globally asymptotically stable.

Assume $\left\{x_{i}(t)\right\}_{i \in[1, m]}^{t \in R^{+}}$is positive solution of systems (6) and (7), and we can establish the following result. 
Theorem 3. The unique positive equilibrium $E^{*}\left(x_{1}^{*}, x_{2}^{*}, \ldots\right.$, $\left.x_{n}^{*}, u_{1}^{*}, u_{2}^{*}, \ldots, u_{n}^{*}\right)$ of systems (6) and (7) is globally asymptotically stable.

Proof. Let

$$
V_{i}\left(x_{i}, u_{i}\right)=x_{i}-x_{i}^{*}-x_{i}^{*} \ln \frac{x_{i}}{x_{i}^{*}}+\frac{b}{2 e}\left(u_{i}-u_{i}^{*}\right)^{2} .
$$

Differentiating $V_{i}$ along the solution of system (6) and using the equilibrium equations, we obtain

$$
\begin{aligned}
V_{i}^{\prime} & =\left(x_{i}-x_{i}^{*}\right)\left(r-a x_{i}-b u_{i}+D \frac{\nabla^{2} x_{i-1}}{x_{i}}\right)+\frac{b}{e}\left(u_{i}-u_{i}^{*}\right)\left(-\eta u_{i}(t)+e x_{i}(t)\right) \\
& =\left(x_{i}-x_{i}^{*}\right)\left(-a\left(x_{i}-x_{i}^{*}\right)-b\left(u_{i}-u_{i}^{*}\right)+D\left(\frac{\nabla^{2} x_{i-1}}{x_{i}}-\frac{\nabla^{2} x_{i-1}^{*}}{x_{i}^{*}}\right)\right)+\frac{b}{e}\left(u_{i}-u_{i}^{*}\right)\left(-\eta\left(u_{i}-u_{i}^{*}\right)+e\left(x_{i}-x_{i}^{*}\right)\right) \\
& =-a\left(x_{i}-x_{i}^{*}\right)^{2}-\frac{b \eta}{e}\left(u_{i}-u_{i}^{*}\right)^{2}+D\left(x_{i}-x_{i}^{*}\right)\left(\frac{\nabla^{2} x_{i-1}}{x_{i}}-\frac{\nabla^{2} x_{i-1}^{*}}{x_{i}^{*}}\right) \leq D\left(x_{i}-x_{i}^{*}\right)\left(\frac{\nabla^{2} x_{i-1}}{x_{i}}-\frac{\nabla^{2} x_{i-1}^{*}}{x_{i}^{*}}\right) .
\end{aligned}
$$

Set

$$
V\left(x_{1}, u_{1}, x_{2}, u_{2}, \ldots, x_{n}, u_{n}\right)=\sum_{i=1}^{n} c_{i} V_{i}\left(x_{i}, u_{i}\right)
$$

$$
\begin{aligned}
V^{\prime}\left(x_{1}, u_{1}, x_{2}, u_{2}, \ldots, x_{n}, u_{n}\right) & =\sum_{i=1}^{n} c_{i} V_{i}^{\prime}\left(x_{i}, u_{i}\right) \\
& \leq \sum_{i=1}^{n} c_{i} D\left(x_{i}-x_{i}^{*}\right)\left(\frac{\nabla^{2} x_{i-1}}{x_{i}}-\frac{\nabla^{2} x_{i-1}^{*}}{x_{i}^{*}}\right) \\
& =D \sum_{i=1}^{n} c_{i}\left(x_{i}-x_{i}^{*}\right)\left(\frac{x_{i+1}+x_{i-1}}{x_{i}}-\frac{x_{i+1}^{*}+x_{i-1}^{*}}{x_{i}^{*}}\right) \\
& =D \sum_{i=1}^{n-1} c_{i} x_{i+1}^{*}\left(\frac{x_{i+1}}{x_{i+1}^{*}}-\frac{x_{i}}{x_{i}^{*}}-\frac{x_{i}^{*} x_{i+1}}{x_{i} x_{i+1}^{*}}+1\right)+D \sum_{i=2}^{n} c_{i} x_{i-1}^{*}\left(\frac{x_{i-1}}{x_{i-1}^{*}}-\frac{x_{i}}{x_{i}^{*}}-\frac{x_{i}^{*} x_{i-1}}{x_{i} x_{i-1}^{*}}+1\right) .
\end{aligned}
$$

It is clear that $\ln \left(x_{i} / x_{i}^{*}\right)-\ln \left(x_{i+1} / x_{i+1}^{*}\right)+\ln \left(x_{i}^{*} x_{i+1} / \quad x_{i} x_{i-1}^{*}\right)=0$ are true. And $1-x+\ln x \leq 0$, for $x>0$, and the $\left.x_{i} x_{i+1}^{*}\right)=0$, and $\ln \left(x_{i} / x_{i}^{*}\right)-\ln \left(x_{i-1} / x_{i-1}^{*}\right)+\ln \left(x_{i}^{*} x_{i-1} / \quad\right.$ equality holds if and only if $x=1$. We have

$$
\begin{aligned}
V^{\prime}\left(x_{1}, u_{1}, x_{2}, u_{2}, \ldots, x_{n}, u_{n}\right) \leq & D \sum_{i=1}^{n-1} c_{i} x_{i+1}^{*}\left(\frac{x_{i+1}}{x_{i+1}^{*}}-\frac{x_{i}}{x_{i}^{*}}-\frac{x_{i}^{*} x_{i+1}}{x_{i} x_{i+1}^{*}}+1\right)+D \sum_{i=2}^{n} c_{i} x_{i-1}^{*}\left(\frac{x_{i-1}}{x_{i-1}^{*}}-\frac{x_{i}}{x_{i}^{*}}-\frac{x_{i}^{*} x_{i-1}}{x_{i} x_{i-1}^{*}}+1\right) \\
= & D \sum_{i=1}^{n-1} c_{i} x_{i+1}^{*}\left(\frac{x_{i+1}}{x_{i+1}^{*}}-\frac{x_{i}}{x_{i}^{*}}-\frac{x_{i}^{*} x_{i+1}}{x_{i} x_{i+1}^{*}}+1+\ln \frac{x_{i}}{x_{i}^{*}}-\ln \frac{x_{i+1}}{x_{i+1}^{*}}+\ln \frac{x_{i}^{*} x_{i+1}}{x_{i} x_{i+1}^{*}}\right) \\
& +D \sum_{i=2}^{n} c_{i} x_{i-1}^{*}\left(\frac{x_{i-1}}{x_{i-1}^{*}}-\frac{x_{i}}{x_{i}^{*}}-\frac{x_{i}^{*} x_{i-1}}{x_{i} x_{i-1}^{*}}+1+\ln \frac{x_{i}}{x_{i}^{*}}-\ln \frac{x_{i-1}}{x_{i-1}^{*}}+\ln \frac{x_{i}^{*} x_{i-1}}{x_{i} x_{i-1}^{*}}\right) \\
\leq & D \sum_{i=1}^{n-1} c_{i} x_{i+1}^{*}\left(\frac{x_{i+1}}{x_{i+1}^{*}}-\frac{x_{i}}{x_{i}^{*}}+\ln \frac{x_{i}}{x_{i}^{*}}-\ln \frac{x_{i+1}}{x_{i+1}^{*}}\right)+D \sum_{i=2}^{n} c_{i} x_{i-1}^{*}\left(\frac{x_{i-1}}{x_{i-1}^{*}}-\frac{x_{i}}{x_{i}^{*}}+\ln \frac{x_{i}}{x_{i}^{*}}-\ln \frac{x_{i-1}}{x_{i-1}^{*}}\right) \\
= & D \sum_{i=1}^{n-1}\left[\left(c_{i} x_{i+1}^{*}-c_{i+1} x_{i}^{*}\right) \frac{x_{i+1}}{x_{i+1}^{*}}+\left(c_{i+1} x_{i}^{*}-c_{i} x_{i+1}^{*}\right) \frac{x_{i}}{x_{i}^{*}}\right] \\
& -D \sum_{i=1}^{n-1}\left[\left(c_{i} x_{i+1}^{*}-c_{i+1} x_{i}^{*}\right) \ln \frac{x_{i+1}}{x_{i+1}^{*}}+\left(c_{i+1} x_{i}^{*}-c_{i} x_{i+1}^{*}\right) \ln \frac{x_{i}}{x_{i}^{*}}\right] .
\end{aligned}
$$




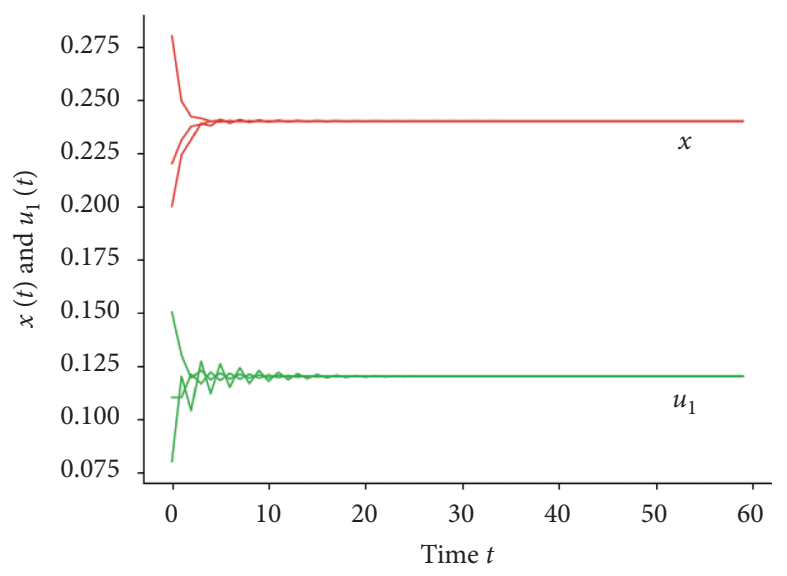

(a)

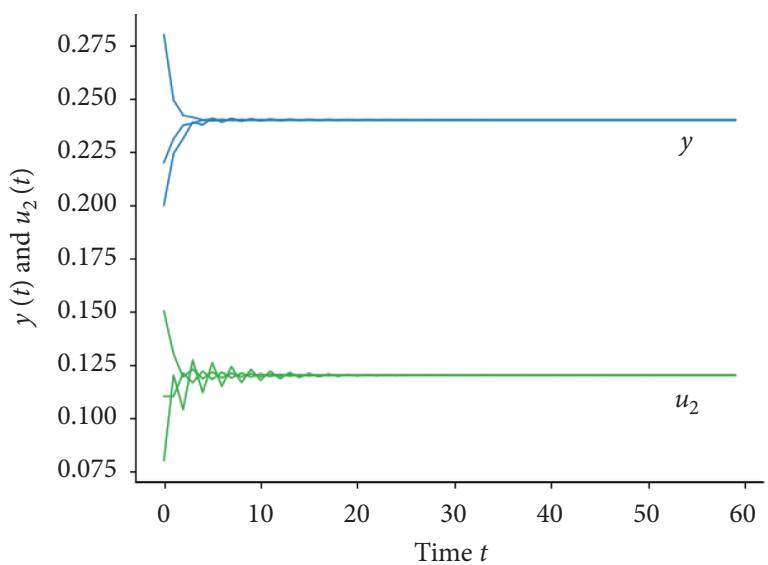

(b)

Figure 1: Dynamic behaviors of system (29) with the initial conditions $(0.28,0.28,0.08,0.08),(0.22,0.22,0.11,0.11)$, and $(0.20 .0 .20,0.08$, $0.08)$ when $r=2.1, a=2 . b=1, e=1, \eta=2$, and $D=1.5$.

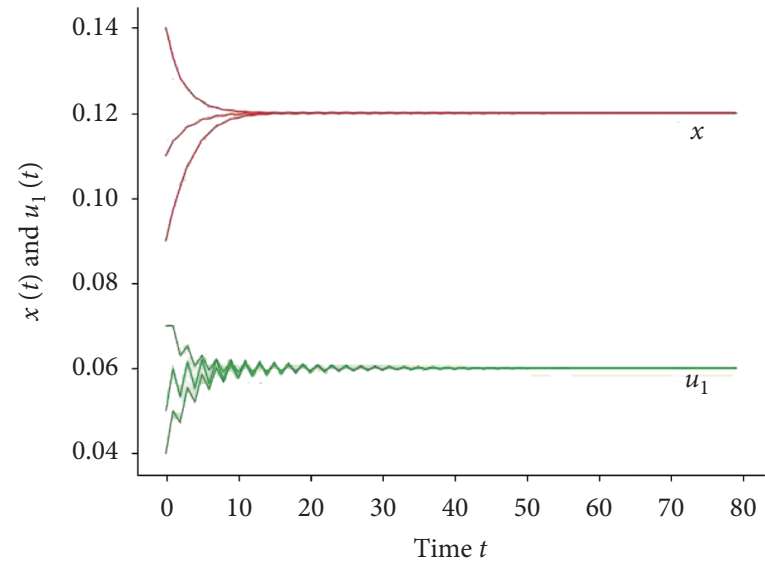

(a)

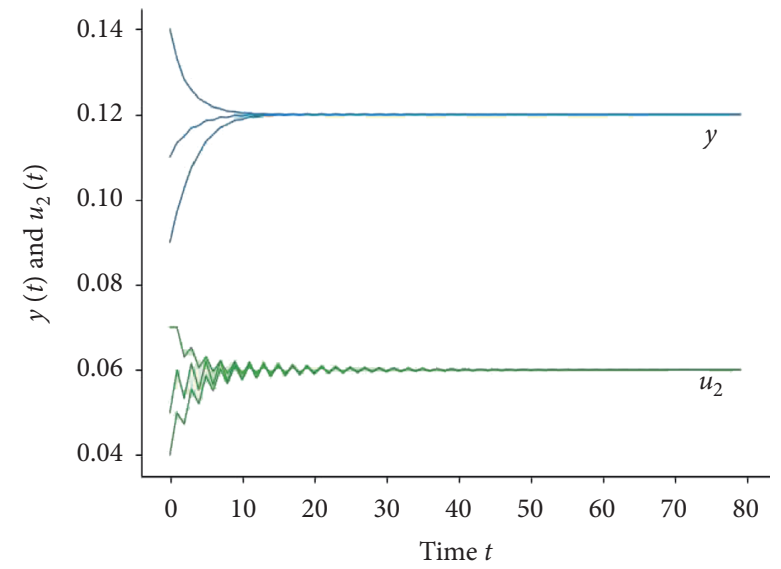

(b)

Figure 2: Dynamic behaviors of system (29) with the initial conditions $(0.11,0.11,0.05,0.05),(0.14,0.14,0.07,0.07)$, and $(0.09 .0 .09,0.04$, $0.04)$ when $r=2.1, a=2 . b=1, e=1, \eta=2$, and $D=1.8$.

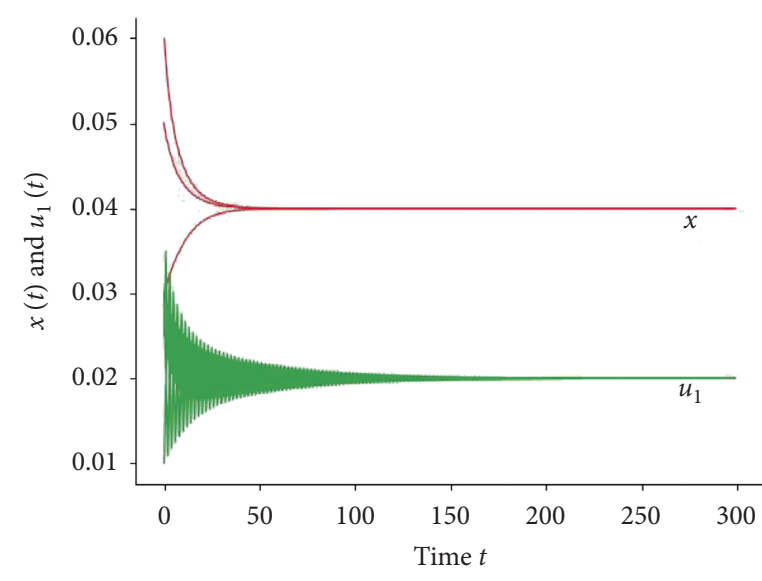

(a)

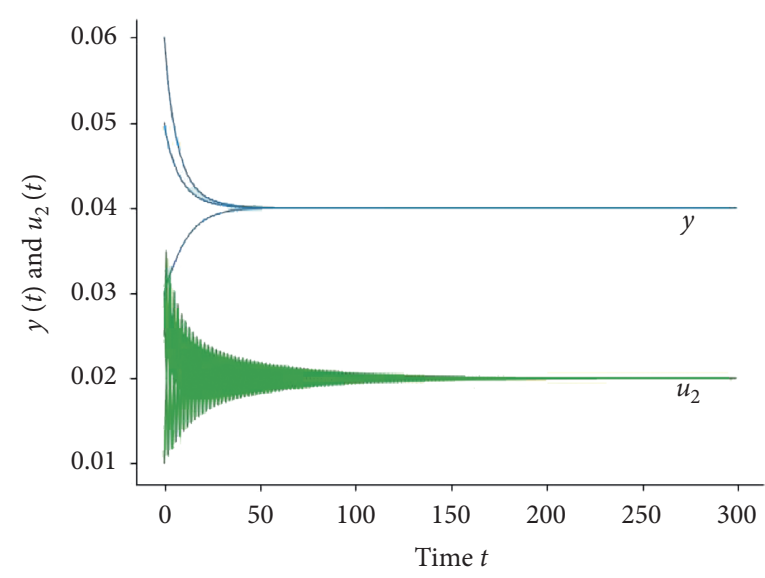

(b)

Figure 3: Dynamic behaviors of system (29) with the initial conditions $(0.03,0.03,0.01,0.01),(0.05,0.05,0.03,0.03)$, and $(0.06 .0 .06,0.025$, 0.025 ) when $r=2.1, a=2 . b=1, e=1, \eta=2$, and $D=2$. 


$$
\begin{aligned}
& \text { If }\left(c_{i+1} / c_{i}\right)=\left(x_{i+1}^{*} / x_{i}^{*}\right), i=1,2, \ldots, n-1, \text { then } \\
& D \sum_{i=1}^{n-1}\left[\left(c_{i} x_{i+1}^{*}-c_{i+1} x_{i}^{*}\right) \frac{x_{i+1}}{x_{i+1}^{*}}+\left(c_{i+1} x_{i}^{*}-c_{i} x_{i+1}^{*}\right) \frac{x_{i}}{x_{i}^{*}}\right] \\
& -D \sum_{i=1}^{n-1}\left[\left(c_{i} x_{i+1}^{*}-c_{i+1} x_{i}^{*}\right) \ln \frac{x_{i+1}}{x_{i+1}^{*}}+\left(c_{i+1} x_{i}^{*}-c_{i} x_{i+1}^{*}\right) \ln \frac{x_{i}}{x_{i}^{*}}\right]=0,
\end{aligned}
$$

and $V^{\prime}\left(x_{1}, u_{1}, x_{2}, u_{2}, \ldots, x_{n}, u_{n}\right) \leq 0$. Through proper selection of $\left\{c_{i}\right\}_{i=1}^{n-1},\left(c_{i+1} / c_{i}\right)=\left(x_{i+1}^{*} / x_{i}^{*}\right)$ can always hold. The proof is finished.

\section{Numerical Example}

In the following example, we will show the feasibility of our main results and discuss the effects of feedback controls. Taking $n=2$ in system (6), we obtain a model with feedback controls between two patches as follows:

$$
\left\{\begin{array}{l}
\frac{\mathrm{d} x}{\mathrm{~d} t}=x\left(r-a x-b u_{1}\right)+D(y-2 x) \\
\frac{\mathrm{d} y}{\mathrm{~d} t}=y\left(r-a y-b u_{2}\right)+D(x-2 y) \\
\frac{\mathrm{d} u_{1}}{\mathrm{~d} t}=-\eta u_{1}(t)+e x(t) \\
\frac{\mathrm{d} u_{2}}{\mathrm{~d} t}=-\eta u_{2}(t)+e y(t)
\end{array}\right.
$$

Its equilibrium equations can be given as follows:

$$
\left\{\begin{array}{l}
x\left(r-a x-b u_{1}\right)+D(y-2 x)=0 \\
y\left(r-a y-b u_{2}\right)+D(x-2 y)=0 \\
-\eta u_{1}+e x=0 \\
-\eta u_{2}+e y=0
\end{array}\right.
$$

which has only a unique positive equilibrium:

$$
E^{*}\left(x^{*}, y^{*}, u_{1}^{*}, u_{2}^{*}\right)=\left(\frac{(r-D) \eta}{a \eta+b e}, \frac{(r-D) \eta}{a \eta+b e}, \frac{(r-D) e}{a \eta+b e}, \frac{(r-D) e}{a \eta+b e}\right),
$$

with $r>4 D \sin ^{2}(\pi / 2(2+1))=D$.

To illustrate our purposes, the parameter values are chosen as follows (the choice of parameter values is hypothetical with appropriate units and not based on data): $r=2.1, a=2, b=1, D=1.5, e=1$, and $\eta=2$; then, there is only a unique positive equilibrium $E^{*}\left(x^{*}, y^{*}, u_{1}^{*}, u_{2}^{*}\right)=$ $(0.24,0.24,0.12,0.12)$. It is easy to see that the conditions in Theorems 2 and 3 are verified. Dynamic behaviors of system (29) with the different initial conditions can be shown in Figure 1. The simulation can illustrate the fact that the positive equilibrium is globally asymptotically stable.

To explore clearly the dynamical behavior of system (29), we investigate the effect of diffusion parameter $D$ by keeping other parameters of the system fixed. Figure 2 exhibits in detail an interesting situation when $D=1.8(r=2.1, a=2$, $b=1, e=1, \eta=2)$, in which the solutions of $u_{1}(t)$ and $u_{2}(t)$ oscillate more obviously and converge more slowly than Figure 1. With further increase of $D$, similar fact will emerge, as depicted in Figure 3.

\section{Conclusions}

To summarize, we have proposed a continuous time but the discrete space logistic model with feedback control and the discrete Dirichlet boundary conditions. By means of the suband supper-solution method and eigenvalue theory, the sufficient conditions of uniqueness and existence of positive steady state solution have been obtained. By constructing Lyapunov functions, global stability of the positive equilibria has been tackled. An example is worked out to illustrate the theoretical results.

It is well known that noise disturbance is unavoidable in real systems and it has an important effect on the stability of systems. And the noise, which can be used to stabilize a given unstable system or to make a system even more stable when the system is already stable, which reveals that the stochastic feedback control, can stabilize and destabilize the deterministic systems $[25,26]$. Therefore, it will be interesting and challenging to investigate stabilization or destabilization of the nonlinear semidiscrete system with feedback control in our future work.

\section{Data Availability}

No data were used to support this study.

\section{Conflicts of Interest}

The authors declare that there are no conflicts of interest regarding the publication of this paper.

\section{Acknowledgments}

This work was supported by Tianjin Municipal Education Commission Scientific Research Project of China (Grant no. 161082) and Applied Study Program (Grant nos. 171006901B, 60204, and WH18012).

\section{References}

[1] R. M. May and G. F. Oster, "Bifurcations and dynamic complexity in simple ecological models," The American Naturalist, vol. 110, no. 974, pp. 573-599, 1976.

[2] B. S. Lalli, J. S. Yu, and M. Chen, "Feedback regulations of a logistic growth," Dynamic Systems and Applications, vol. 5, pp. 117-124, 1996.

[3] G. S. Ladde and S. Sathananthan, "Stability of lotka-volterra model," Mathematical and Computer Modelling, vol. 16, no. 3, pp. 99-107, 1992.

[4] D. Ricardo da Costa, R. O. Medrano-T, and E. D. Leonel, "Route to chaos and some properties in the boundary crisis of a generalized logistic mapping," Physica A: Statistical Mechanics and its Applications, vol. 486, pp. 674-680, 2017. 
[5] Q. Lin, "Stability analysis of a single species logistic model with Allee effect and feedback control," Advances in Difference Equations, vol. 2018, no. 1, 2018.

[6] A. M. Turing, "The chemical basis of morphogenesis," PhiloSophical Transactions of the Royal Society B: Biological Sciences, vol. 237, no. 641, pp. 37-72, 1952.

[7] L. Xu, L. J. Zhao, Z. X. Chang, J. T. Feng, and G. Zhang, "Turing instability and pattern formation in a semi-discrete brusselator model," Modern Physics Letters B, vol. 27, no. 1, pp. 1350006-1350011, 2013.

[8] D. Pal and G. P. Samanta, "Effects of dispersal speed and strong Allee effect on stability of a two-patch predator-prey model," International Journal of Dynamics and Control, vol. 6, no. 4, pp. 1484-1495, 2018.

[9] D. Jana and G. P. Samanta, "Significance of prey harvesting in prey-predator system in discrete time scale using interval parameters," International Journal of Ecological Economics and Statistics, vol. 39, no. 2, pp. 46-60, 2018.

[10] L. Xu, G. Zhang, and J. F. Ren, "Turing instability for a two dimensional semi-discrete Oregonator model," Wseas Transactions on Mathematics, vol. 10, pp. 201-209, 2011.

[11] F. X. Mai, L. J. Qin, and G. Zhang, "Turing instability for a semi-discrete Gierer-Meinhardt system," Physica A: Statistical Mechanics and Its Applications, vol. 391, no. 5, pp. 2014-2022, 2012.

[12] L. Chen and J. Sun, "Global stability of an SI epidemic model with feedback controls," Applied Mathematics Letters, vol. 28, pp. 53-55, 2014.

[13] K. Gopalsamy and P. X. Weng, "Feedback regulation of logistic growth," International Journal of Mathematics and Mathematical Sciences, vol. 16, no. 1, pp. 177-192, 1993.

[14] L. Xu, S. S. Lou, P. Q. Xu, and G. Zhang, "Feedback control and parameter invasion for a discrete competitive Lotka-Volterra system," Discrete Dynamics in Nature and Society, vol. 2018, Article ID 7473208, 8 pages, 2018.

[15] H.-F. Huo and W.-T. Li, "Positive periodic solutions of a class of delay differential system with feedback control," Applied Mathematics and Computation, vol. 148, no. 1, pp. 35-46, 2004.

[16] K. Gopalsamy, Stability and Oscillations in Delay Differential Equations of Population Dynamics, Kluwer Academic, Boston, MA, USA, 1992.

[17] Y.-H. Fan and L.-L. Wang, "Global asymptotical stability of a Logistic model with feedback control," Nonlinear Analysis: Real World Applications, vol. 11, no. 4, pp. 2686-2697, 2010.

[18] S. Saha and G. P. Samanta, "Influence of dispersal and strong Allee effect on a two-patch predator-prey model," International Journal of Dynamics and Control, vol. 7, no. 4, pp. 1321-1349, 2019.

[19] A. Al-Saffar and E.-j. Kim, "Sustainable theory of a logistic model-Fisher information approach," Mathematical Biosciences, vol. 285, pp. 81-91, 2017.

[20] M. T. Hoang and A. M. Nagy, "Uniform asymptotic stability of a Logistic model with feedback control of fractional order and nonstandard finite difference schemes," Chaos, Solitons \& Fractals, vol. 123, pp. 24-34, 2019.

[21] M. Y. Li and Z. Shuai, "Global-stability problem for coupled systems of differential equations on networks," Journal of Differential Equations, vol. 248, no. 1, pp. 1-20, 2010.

[22] H.-L. Li, L. Zhang, Z. Teng, Y.-L. Jiang, and A. Muhammadhaji, "Global stability of an SI epidemic model with feedback controls in a patchy environment," Applied Mathematics and Computation, vol. 321, pp. 372-384, 2018.
[23] L. Meng, X. Li, and G. Zhang, "Simple diffusion can support the pitchfork, the flip bifurcations, and the chaos," Communications in Nonlinear Science and Numerical Simulation, vol. 53, pp. 202-212, 2017.

[24] X. F. Li and G. Zhang, "Positive solutions of a general discrete dirichlet boundary value problem," Discrete Dynamics in Nature and Society, vol. 2016, Article ID 7456937, 7 pages, 2016.

[25] X. Mao, "Almost sure exponential stabilization by discretetime stochastic feedback control," IEEE Transactions on Automatic Control, vol. 61, pp. 1619-1624, 2016.

[26] Q. Zhu and T. Huang, "Stability analysis for a class of stochastic delay nonlinear systems driven by G-Brownian motion," Systems \& Control Letters, vol. 140, 2020. 